\title{
Modelled effects of temperature gradients and waves on the hydroxyl rotational distribution in ground-based airglow measurements
}

\author{
Christoph Franzen ${ }^{1,2}$, Patrick Joseph Espy ${ }^{1,2}$, and Robert Edward Hibbins ${ }^{1,2}$ \\ ${ }^{1}$ Norwegian University of Science and Technology (NTNU), Trondheim, 7491, Norway \\ ${ }^{2}$ Birkeland Centre for Space Science (BCSS), Department of Physics and Technology, University of Bergen, Norway
}

Correspondence: Christoph Franzen (franzen.christoph@rwth-aachen.de)

Received: 1 April 2019 - Discussion started: 3 April 2019

Revised: 4 November 2019 - Accepted: 18 November 2019 - Published: 9 January 2020

\begin{abstract}
Spectroscopy of the hydroxyl $(\mathrm{OH})$ airglow has been a commonly used way to remotely sense temperatures in the mesopause region for many decades. This technique relies on the $\mathrm{OH}$ rotational state populations to be thermalized through collisions with the surrounding gas into a Boltzmann distribution characterized by the local temperature. However, deviations of the rotational populations from a Boltzmann distribution characterized by a single temperature have been observed and attributed to an incomplete thermalization of the $\mathrm{OH}$ from its initial, non-thermodynamicequilibrium distribution. Here we address an additional cause for the apparent amount of excess population in the higher rotational levels of the $\mathrm{OH}$ airglow brought about by integrating these $\mathrm{OH}$ emissions through vertical gradients in the atmospheric temperature. We find that up to $40 \%$ of the apparent excess population, currently attributed to incomplete thermalization, can be due to the vertical temperature gradients created by waves. Additionally, we find that the populations of the different upper vibrational levels are affected differently. These effects need to be taken into account in order to assess the true extent of non-thermodynamic-equilibrium effects on the $\mathrm{OH}$ rotational populations.
\end{abstract}

\section{Introduction}

The hydroxyl $(\mathrm{OH})$ airglow has been employed for many years for remote sensing of the mesosphere and lower thermosphere (MLT) region, an example of which may be found in Smith et al. (2010). The $8 \mathrm{~km}$ thick airglow layer is cre- ated at about $90 \mathrm{~km}$ altitude (Baker and Stair, 1988; Xu et al., 2012) by the highly exothermic reduction of ozone:

$\mathrm{H}+\mathrm{O}_{3} \rightarrow \mathrm{OH}^{*}+\mathrm{O}_{2}\left(5.3 \times 10^{-19} \mathrm{~J}\right)$.

The excess heat of reaction, $\sim 5.3 \times 10^{-19} \mathrm{~J}$, produces the $\mathrm{OH}^{*}$ in excited vibrational quantum levels of $v^{\prime}=6-9$ (e.g. Mlynczak and Solomon, 1993). Subsequent radiative cascading and collisional-deactivation produces $\mathrm{OH}^{*}$ in all vibrational levels $\leq 9$. Radiative deactivation can occur between any two vibrational quantum levels, but transitions with $\Delta v=2$ are preferred (Langhoff et al., 1986).

The excess energy of Reaction (1) also creates rotational excitation within the $\mathrm{OH}^{*}$ molecule in addition to the vibrational excitation. The nascent rotational population for high rotational levels, $J^{\prime}$, shows a distribution characteristic of a temperature far above the local atmospheric temperature. Llewellyn et al. (1978) reported a nascent temperature of $760 \mathrm{~K}$ for $v^{\prime}=9$, whereas others report temperatures as high as 9000-10000 K (Oliva et al., 2015; Kalogerakis et al., 2018). Low rotational levels (with $N \leq 4$ ) with energy separations less than $\mathrm{kT}$, the amount typically exchanged during collisions, have been observed to have efficient energy transfer in the thermalization process (Maylotte et al., 1972; Polanyi and Sloan, 1975; Polanyi and Woodall, 1972). Thus, emission from these states has been observed to be characterized by a single-temperature Boltzmann distribution (Harrison et al., 1970, 1971; Pendleton et al., 1993; Perminov et al., 2007; Sivjee et al., 1972; Sivjee and Hamwey, 1987). However, emission observed from the higher rotational levels $(N>4)$, where the energy separation exceeds $\mathrm{kT}$, has in- 
dicated an anomalous, non-thermalized population that cannot be described using the same Boltzmann temperature that characterizes the lower rotational levels (Cosby and Slanger, 2007; Dodd et al., 1994; Kalogerakis, 2019; Noll et al., 2015; Pendleton et al., 1989, 1993). In keeping with the terminology employed by Pendleton et al. (1993), we refer to the condition where the population can be described by a singletemperature Boltzmann distribution as local thermodynamic equilibrium (LTE), whereas non-local thermodynamic equilibrium (NLTE) is used when the population departs from that distribution. Work is currently underway to use observations of the excess populations in the high rotational levels of the $\mathrm{OH}$ airglow to determine state-to-state quenching coefficients and to understand the thermalization process in $\mathrm{OH}$ (Kalogerakis et al., 2018). The term LTE as used here is not technically correct as it does not account for radiative effects (i.e. the emission of airglow photons) on the rotationalvibrational level population distribution. Instead it relates only to the collisional distribution of the rotational levels being characterized by the temperature of the surrounding gas, as has been done in Pendleton et al. (1993).

Here we examine the effects of temperature gradients in the $\mathrm{OH}$ emission region on the resulting vertically integrated spectrum of the Meinel $\Delta v=2$ sequence. To achieve this, model work was executed, where the model assumes that for each vibrational level, the rotational population distribution of the $\mathrm{OH}$ is in LTE at every altitude. The emission in each rotational line is then integrated vertically. We find that, even if the $\mathrm{OH}$ rotational levels are in strict LTE with the surrounding atmosphere, the temperature gradients through the $\mathrm{OH}$ emission region will create apparent excess emission in the higher $\mathrm{OH}$ rotational lines. Here we calculate the apparent excess population relative to the Boltzmann population expected using the temperature determined by the population of rotational levels with $N \leq 4$. This excess population can be incorrectly interpreted as due to NLTE effects, affecting the subsequent calculations of the thermalization process. The deviations in the inferred populations from a singletemperature Boltzmann distribution are compared with observations that include both NLTE and temperature gradient effects. This comparison is made for realistic atmospheric temperature profiles that have been perturbed with realistic atmospheric gravity waves in order to help quantify the true NLTE content needed to construct a quantitative picture of $\mathrm{OH}$ thermalization. Recent measurements indicate that the Boltzmann distribution of the rotational levels may be characterized by a temperature that is higher than that of the surrounding gas due to incomplete thermalization (Noll et al., 2018). However, the purpose of this paper is to show that, even if complete thermalization with the surrounding gas takes place, ground-based measurements integrating through temperature gradients within the $\mathrm{OH}$ layer will not see a rotational population described by a Boltzmann distribution characterized by a single temperature.

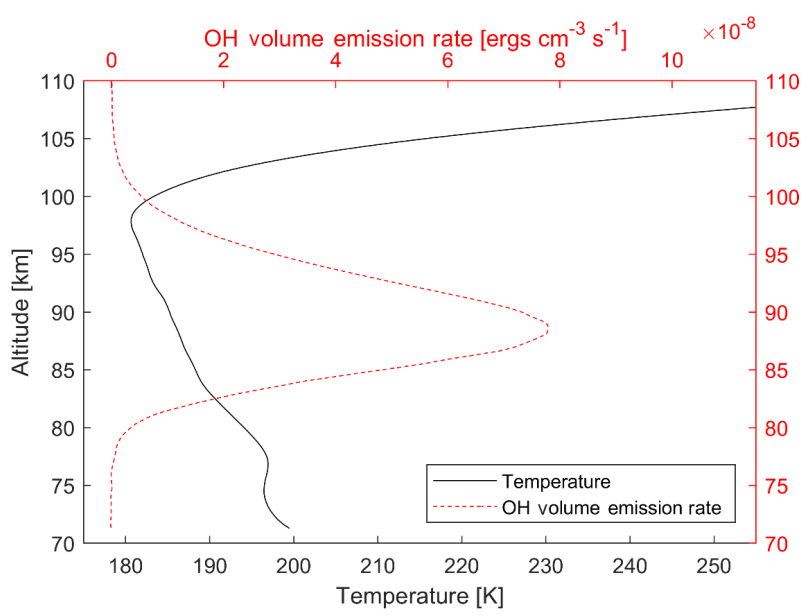

Figure 1. Example of a typical temperature profile (black line) of the airglow layer and the VER (dashed red line) from the SABER satellite showing the variation of temperature through the emission region. This specific measurement is a zonal mean and monthly average from July 2016 between the latitudes of 20 and $30^{\circ} \mathrm{N}$ for the $\Delta v=2$ bands from the upper-state vibrational levels 8 and 9. An erg is equivalent to $10^{-7} \mathrm{~J}$.

\section{Overview of the $\mathrm{OH}$ emission}

Figure 1 shows an example of the $\mathrm{OH}$ airglow volume emission rate (VER) and a temperature profile, measured from the Sounding of the Atmosphere using Broadband Emission Radiometry (SABER) instrument aboard the NASA Thermosphere Ionosphere Mesosphere Energetics and Dynamics (TIMED) satellite (Mlynczak, 1997; Russell et al., 1999). This specific measurement is a zonal mean and monthly average from July 2016 between the latitudes of 20 and $30^{\circ} \mathrm{N}$. The OH VER from the vibrational levels 8 and 9 is shown in red in the figure, while the black curve shows the temperature profile. It can be seen that the temperature is not constant through the $\mathrm{OH}$ layer. In this example the atmospheric temperature changes by over $10 \mathrm{~K}$ through the layer. This observed behaviour is similar to other observations, for example from French and Mulligan (2010), who compared TIMED/SABER observations with ground-based observations.

Waves will exacerbate this effect by perturbing both the OH VER and changing the temperature gradient. Thus, the rotational level population distribution of the $\mathrm{OH}$, even if thermalized at each altitude, will have different temperatures at each of those altitudes. Any instrument that integrates through the $\mathrm{OH}$ layer will therefore not see rotational line emission resulting from a single, average temperature but from the whole span of temperatures present in the layer. 


\section{Method}

We utilize a steady-state model of the OH VER, described below, to synthesize individual synthetic rotational spectra at $1 \mathrm{~km}$ intervals from 74 to $110 \mathrm{~km}$. The model assumes that for each vibrational level, the rotational level population distribution of the $\mathrm{OH}$ is in LTE (i.e. can be described by a single-temperature Boltzmann distribution) with the local temperature at each altitude. Each rotational line is integrated in altitude to give the net spectrum that would be observed by an instrument integrating through the layer. The distribution of emission in the rotational lines is then used to infer the population of the $\mathrm{OH}$ rotational levels, allowing us to quantify the portion of the inferred excess population in the upper levels that is due to the temperature gradients across the $\mathrm{OH}$ layer.

\subsection{The OH steady-state model}

The atmospheric background temperature and concentrations of $\mathrm{N}_{2}, \mathrm{O}_{2}, \mathrm{H}$ and $\mathrm{O}$ are taken from the US Naval Research Laboratory's mass spectrometer and incoherent scatter radar model (NRLMSISE-00) (Picone et al., 2002). The steadystate ozone concentration is then calculated from balancing the production and loss processes. The production mechanism is

$\mathrm{O}+\mathrm{O}_{2}+\mathrm{M} \rightarrow \mathrm{O}_{3}+\mathrm{M}$

where $\mathrm{M}$ is a reaction mediator. The temperature-dependent rate coefficients for this reactions are taken from the International Union for Pure and Applied Chemistry (IUPAC) Gas Kinetic Database (Atkinson et al., 2004). Loss processes include losses due to $\mathrm{O}$ via

$\mathrm{O}+\mathrm{O}_{3} \rightarrow 2 \mathrm{O}_{2}$,

using the reaction rate coefficient of Sander et al. (2003). The loss of $\mathrm{O}_{3}$ to atomic hydrogen,

$\mathrm{H}+\mathrm{O}_{3} \rightarrow \mathrm{OH}^{*}+\mathrm{O}$

was also used to calculate the production rate of $\mathrm{OH}^{*}$ for each vibrational level, using the reaction rate coefficient (Sander et al., 2003). Due to the exothermicity of Reaction (4), vibrational levels from $v^{\prime}=6$ to 9 can be populated. The production of each vibrational level $\mathrm{OH}^{*}\left(\mathrm{v}^{\prime}\right)$ is calculated using the branching ratios from Sander et al. (2019). Collisional loss for each $\mathrm{OH}^{*}\left(v^{\prime}\right)$ vibrational level was calculated for collisions with $\mathrm{O}, \mathrm{O}_{2}$ and $\mathrm{CO}_{2}$ using the rate coefficients of Dodd et al. (1991), Knutsen et al. (1996), Dyer et al. (1997) and Chalamala and Copeland (1993). The model assumes quenching to the ground vibrational state, known as "sudden death" for the $\mathrm{O}$, and stepwise quenching by one vibrational unit for the $\mathrm{O}_{2}$ and the $\mathrm{CO}_{2}$ (McDade and Llewellyn, 1987). $\mathrm{N}_{2}$ is not considered as a quencher in this model. The rate coefficient for $\mathrm{OH}$ quenching with $\mathrm{N}_{2}$ is small, and Knutsen et al. (1996) were only able to provide an upper limit. Since the $\mathrm{O}_{2} / \mathrm{N}_{2}$ mixing ratio is nearly constant up to the turbopause, the $\mathrm{O}_{2}$ is 3 times more effective at quenching than the $\mathrm{N}_{2}$. Thus, neglecting the $\mathrm{N}_{2}$ is well within the uncertainty of the $\mathrm{O}_{2}$ rate coefficient and does not significantly affect the altitude distribution of the $\mathrm{OH}$. The relative shape and peak height of the altitude profiles of the individual vibrational levels agree closely with those of the more sophisticated model of Adler-Golden (1997). As previously mentioned, the rotational population distribution within this vibrational level is taken to be a Boltzmann distribution characterized by the local temperature at this altitude.

The total radiative loss from each vibrational level is given by $N_{v^{\prime}}(z) \cdot A_{v^{\prime}}$, where $N_{v^{\prime}}(z)$ is the concentration of the hydroxyl $v^{\prime}$ vibrational level at altitude $z$ and $A_{v^{\prime}}$ is its inverse lifetime, calculated from (Langhoff et al., 1986). The total VER of any $v^{\prime}$ to $v^{\prime \prime}$ vibrational transition is then given by $V_{v^{\prime} v^{\prime \prime}}(z)=A_{v^{\prime} v^{\prime \prime}} \cdot N_{v^{\prime}}(z)$, where $A_{v^{\prime} v^{\prime \prime}}$ is the transition probability for the vibrational transition from $v^{\prime}$ to $v^{\prime \prime}$ that is calculated from the Einstein coefficients from Langhoff et al. (1986). Although transition probabilities from a number of different studies differ from those of Langhoff et al. (1986), the integration over the rotational distribution, required to obtain the vibrational population, is relatively insensitive to the choice of transition probabilities. This radiative cascade into lower vibrational levels then acts as an additional production term for levels below $v^{\prime}=9$. Balancing these production and loss terms at each height yields the steady-state concentration of each $\mathrm{OH}^{*}$ vibrational level as a function of height, $N_{v^{\prime}}(z)$. Such a model has been shown to fit observations, for example TIMED/SABER (Xu et al., 2012). The application of this model to the simulation of a ground-based measurement under the influence of a realistic temperature gradient is undertaken as described below.

\subsection{The wave model}

As mentioned above, the background atmospheric temperature profile can be perturbed by waves. We therefore include wave-induced temperature perturbations in the model. Following Holton (1982), we limited the wave growth with altitude to maintain temperature gradients to below the dry adiabatic lapse rate. The wave has the form

$A_{\mathrm{w}}(z) \cdot \cos \left(\frac{2 \pi z}{\lambda_{\mathrm{w}}}+\varphi_{\mathrm{w}}\right)$,

where $\phi_{\mathrm{w}}$ is the wave's phase, and $A_{\mathrm{w}}(z)$ is a function of altitude so as not to exceed the dry adiabatic lapse rate. The lower edge of the model is at $74 \mathrm{~km}$ altitude. The wave amplitude as a function of altitude is shown in Fig. 2 for the case of a wave with an amplitude of $10 \mathrm{~K}$ at $74 \mathrm{~km}$ altitude and a vertical wavelength of $20 \mathrm{~km}$. This example is given for an isothermal atmosphere of $200 \mathrm{~K}$ (Fig. 2a, dashed black line). The wave grows in amplitude with altitude to conserve en- 


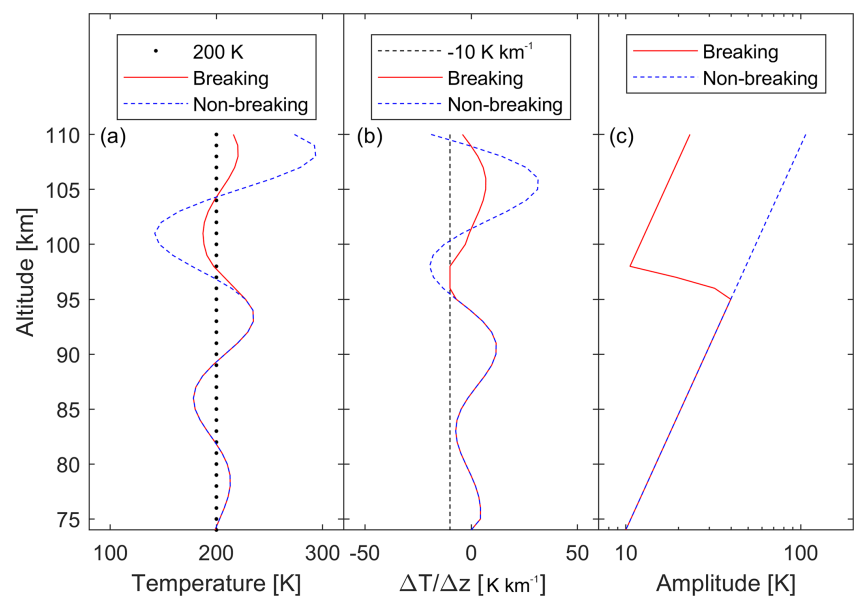

Figure 2. (a) Example of a wave of amplitude $10 \mathrm{~K}$ at $74 \mathrm{~km}$ altitude perturbing an isothermal atmosphere at $T=200 \mathrm{~K}$ (dotted black line). The dashed blue line represents the wave without any breaking, and the red line is the breaking wave as used in the model. (b) The rate of change of temperature with altitude for the wave shown in (a). When the temperature changes faster than $10 \mathrm{~K} \mathrm{~km}^{-1}$ (dashed black line) the wave breaks. (c) At these altitudes, the amplitude of the breaking wave (red line) decreases, while the nonbreaking wave continues to grow exponentially (dashed blue line).

ergy (dashed blue line), but at regions where the lapse rate exceeds the dry adiabatic lapse rate (here between 90 and $95 \mathrm{~km}$, and between 110 and $115 \mathrm{~km}$ ), the wave loses energy and the amplitude decreases (Holton, 1982). The breaking wave is shown in red in Fig. 2a. Figure $2 b$ shows the instantaneous lapse rate (change in temperature with altitude) of the non-breaking wave (dashed blue line) and the breaking wave (red), which never crosses the dry adiabatic lapse rate of $10 \mathrm{~K} \mathrm{~km}^{-1}$ (dashed black line). Figure $2 \mathrm{c}$ shows the amplitude of the non-breaking wave (dashed blue line), which increases exponentially, and for the breaking wave (red), which decreases at the altitudes where the wave dissipates energy.

The background atmosphere mixing ratios from the NRLMSISE-00 model were also perturbed using the gravity wave polarization relations from Vincent (1984). This perturbed background atmosphere and this temperature profile were then used in the steady-state model for the $\mathrm{OH}$ to yield a new, wave-perturbed $N_{v^{\prime}}(z)$ for the analysis.

\subsection{Simulation of a ground-based measurement}

The VER of a rotational transition for the $J^{\prime}$ to $J^{\prime \prime}$ state from the upper state, $v^{\prime}$, of the $v^{\prime}$ to $v^{\prime \prime}$ vibrational band is given by

$V_{v^{\prime} v^{\prime \prime} J^{\prime} J^{\prime \prime}}(z)=N_{v^{\prime}, J^{\prime}}(z) \cdot A_{v^{\prime} v^{\prime \prime}, J^{\prime} J^{\prime \prime}}$,

where $A_{v^{\prime} v^{\prime \prime}, J^{\prime} J^{\prime \prime}}$ is the transition probability for the $J^{\prime}$ to $J^{\prime \prime}$ rotational transition in the $v^{\prime}$ to $v^{\prime \prime}$ vibrational transition. Due to vibrational-rotational coupling, these coefficients are specific to each vibrational transition. $N_{v^{\prime}, J^{\prime}}(z)$ is the population of the upper rotational level, $J^{\prime}$, in the upper vibrational level, $v^{\prime}$. We concentrate in this paper on the $3 / 2$ electronic subset of the $\mathrm{OH}$ airglow and drop the spin-orbit splitting quantum number $F$ in all equations for readability. Assuming that collisions have thermalized the closely spaced rotational levels with the surrounding gas at temperature $T$, their population may be described using a Boltzmann distribution written as

$N_{v^{\prime}, J^{\prime}}(z)=\frac{N_{v^{\prime}}(z) \cdot 2\left(2 J^{\prime}+1\right)}{Q_{\mathrm{R}}(T(z))} \cdot \exp \left(\frac{-E_{v^{\prime}, J^{\prime}}}{k_{\mathrm{B}} \cdot T(z)}\right)$,

where $N_{v^{\prime}}(z)$ is the total population of the $v^{\prime}$ vibrational level at altitude $z$ calculated from the model, $E_{v^{\prime}, J^{\prime}}$ is the energy of the $J^{\prime}$ rotational level, the factor $2\left(2 J^{\prime}+1\right)$ is the degeneracy of that level (including $\Lambda$-doubling) and $Q_{\mathrm{R}}(T(z))$ is the rotational partition function (Herzberg, 1950). $k_{\mathrm{B}}$ is the Boltzmann constant.

Using the rotational transition probabilities of Rothman et al. (2013), the rotational line VERs are calculated within a single $v^{\prime}$ to $v^{\prime \prime}$ transition assuming that the $\mathrm{OH}$ is in LTE for the rotational level populations with the surrounding gas at each altitude and therefore follows a Boltzmann distribution of population characterized by the local temperature. The VER of each rotational line of a given vibrational transition is integrated through the layer from $74 \mathrm{~km}$ through to $110 \mathrm{~km}$ to give the intensity of the line, $I_{v^{\prime} v^{\prime \prime}, J^{\prime} J^{\prime \prime}}$. This results in a net spectrum of low rotational lines whose intensities are enhanced relative to the mean temperature of the emission region when emitted from the cooler regions. Accompanying this are high rotational lines whose intensities are enhanced relative to the mean temperature of the emission region when emitted from the warm regions. Each of these is weighted by the VER of the vibrational transition at each altitude, $N_{v^{\prime}}(z) \cdot A_{v^{\prime} v^{\prime \prime}}$.

As an example, the $\mathrm{OH}$ model was used to create a synthetic spectrum of the $(7,4)$ rotational-vibrational band, assuming LTE for the rotational level populations at every altitude level, for conditions for mid-July for a mid-latitude region. Specifically, the data presented are for Boulder, Colorado $\left(40.0^{\circ} \mathrm{N} ; 105.6^{\circ} \mathrm{W}\right)$, to make a direct comparison with the findings from Pendleton et al. (1993). The background temperature profile (black line) and the $(7,4)$ VER variation with altitude (red line) are shown in Fig. 3a. These are perturbed by the wave with an initial amplitude of $10 \mathrm{~K}$ at $74 \mathrm{~km}$ altitude, which has grown to about $30 \mathrm{~K}$ at $90 \mathrm{~km}$ altitude, and a vertical wavelength of $30 \mathrm{~km}$, shown in the dashed blue line. Waves with similar amplitudes have been observed at these altitudes (Picard et al., 2004).

The resulting distribution of rotational line intensities as a function wavelength for two altitudes is shown in Fig. 3b and c. The net spectrum as observed by a ground-based instrument is analysed, as detailed below, to examine the influence of this high rotational level tail on the fitted temperature. 


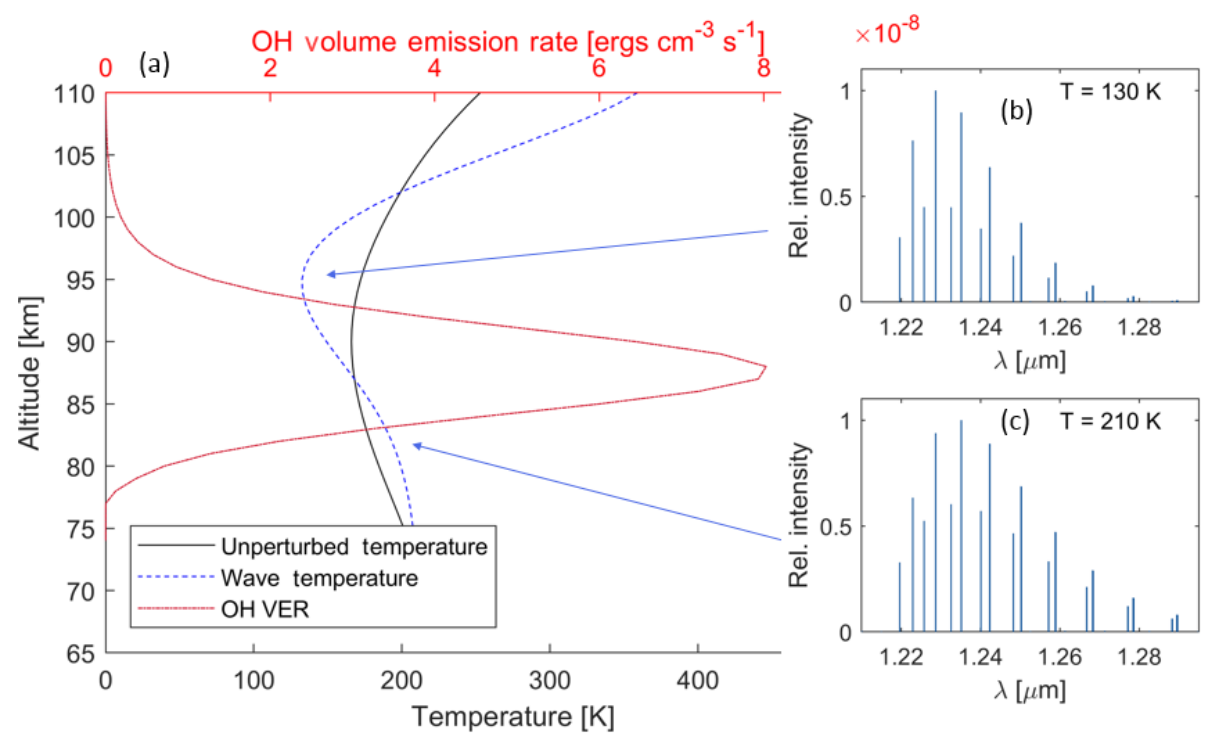

Figure 3. (a) Illustration of the change in the $\mathrm{OH}$ airglow spectrum with altitude due to the temperature profile. The red line shows a modelled VER profile of the $\mathrm{OH}$ in ergs per cubic centimetre per second ( $\mathrm{ergs} \mathrm{cm}^{-3} \mathrm{~s}^{-1}$ ). The black line shows the background temperature profile retrieved from the NRLMSISE-00 model. The dashed blue line shows the same temperature profile but perturbed by a wave with an amplitude which has grown to about $30 \mathrm{~K}$ at $90 \mathrm{~km}$ altitude and a vertical wavelength of $30 \mathrm{~km}$. The insets (b) and (c) on the right show $\mathrm{OH}$ spectra of the $(7,4)$ transition at different altitudes for the wave case. Note that, even though the figure shows the $\mathrm{P}_{1}$ and $\mathrm{P}_{2}$ lines, only the $\mathrm{P}_{1}$ lines are considered in the model. With the example wave given here, the temperature variation increases the VER of the lower rotational lines at $95 \mathrm{~km}$ altitude and of the higher rotational lines at $80 \mathrm{~km}$.

\subsection{Temperature fitting}

After integration through the layer, the relative population of the $J^{\prime}$ state relative to the lowest rotational energy level, $E_{v^{\prime}}$, is now given in terms of the line intensity by

$$
\begin{aligned}
\frac{I_{v^{\prime}, v^{\prime \prime}, J^{\prime} J^{\prime \prime}}}{2\left(2 J^{\prime}+1\right) \cdot A_{v^{\prime} v^{\prime \prime}, J^{\prime} J^{\prime \prime}}} & =Q_{\mathrm{R}}(T) N_{v^{\prime}} \\
& \cdot \exp \left(\frac{-\left(E_{v^{\prime}, J^{\prime}}-E_{v^{\prime}}\right)}{k_{\mathrm{B}} T}\right),
\end{aligned}
$$

where $N v^{\prime}$ is the integral of the vibrational population $N_{v^{\prime}}(z)$ over altitude, and $T$ is the effective rotational temperature of the altitude-integrated spectrum. From this, the observed line intensities from rotational levels of known quantum number, energy, and transition probability may be used to define the relative total population and temperature using

$$
\begin{aligned}
& \ln \left(\frac{I_{v^{\prime}, v^{\prime \prime}, J^{\prime} J^{\prime \prime}}}{2\left(2 J^{\prime}+1\right) \cdot A_{v^{\prime} v^{\prime \prime}, J^{\prime} J^{\prime \prime}}}\right)=\ln \left(Q_{\mathrm{R}}(T) N_{v^{\prime}}\right) \\
& \quad-\frac{1}{T}\left[\frac{\left(E_{v^{\prime}, J^{\prime}}-E_{v^{\prime}}\right)}{k_{\mathrm{B}}}\right] .
\end{aligned}
$$

Figure 4 shows the result in terms of Eq. (9) of integrating each rotational line in the $(7,4)$ Meinel band for the atmospheric perturbation of a wave with an amplitude of $30 \mathrm{~K}$ at $90 \mathrm{~km}$ altitude and $30 \mathrm{~km}$ vertical wavelength. This is the same wave as shown in Fig. 3. Following Pendleton et al. (1993), fitting a temperature to the lowest three rotational levels of the $\mathrm{OH}$ spectrum yields the dashed red curve shown in Fig. 4. It may be seen that the lowest three rotational levels are well characterized by a single, Boltzmann rotational temperature, $T_{1,3}=155.8 \pm 0.8 \mathrm{~K}$, as has been observed in nightglow spectra (Espy and Hammond, 1995; Franzen et al., 2017; Harrison et al., 1970; Noll et al., 2015). However, there is excess emission in the higher rotational lines which could be interpreted as populations exceeding that expected from a thermalized Boltzmann distribution. This apparent excess population occurs even though the $\mathrm{OH}$ distribution was constrained to be a Boltzmann distribution with a single temperature at each altitude.

A non-linear formulation can be used in order to characterize this excess population. This non-linear fit is of the form

$$
\begin{aligned}
& \ln \left(\frac{I_{v^{\prime}, v^{\prime \prime}, J^{\prime} J^{\prime \prime}}}{2\left(2 J^{\prime}+1\right) \cdot A_{v^{\prime} v^{\prime \prime}, J^{\prime} J^{\prime \prime}}}\right)=\ln \left(Q_{\mathrm{R}}(T) N_{v^{\prime}}\right) \\
& -\frac{1}{T}\left[\frac{\left(E_{v^{\prime}, J^{\prime}}-E_{v^{\prime}}\right)}{k_{\mathrm{B}}}\right]+\frac{\beta}{T^{2}}\left[\frac{\left(E_{v^{\prime}, J^{\prime}}-E_{v^{\prime}}\right)}{k_{\mathrm{B}}}\right]^{2} .
\end{aligned}
$$

Here $\beta$ is a non-linearity parameter, which is a free parameter in the non-linear fit. This non-linear fit is also presented in Fig. 4 as a solid blue line, showing that the intensity in rotational lines as high as $J^{\prime}=9.5$ is now fitted. The retrieved temperature, $T_{\mathrm{NL}}=154.9 \pm 0.1 \mathrm{~K}$, is the same as $T_{1,3}$ within the fitting uncertainties, with a $\beta$ factor of 0.019 . 


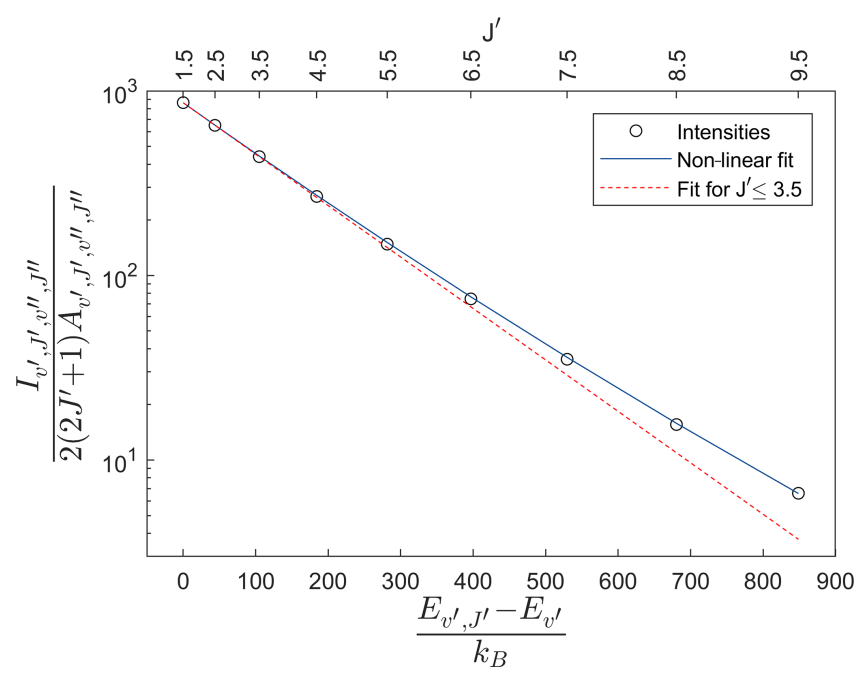

Figure 4. Population of P-branch lines of the $(7,4)$ Meinel band as calculated with an atmospheric background profile containing the wave shown in Fig. 3. The energies given on the $x$ axis are relative to the lowest rotational energy. An exponential fit to the lowest three rotational levels (dashed red line) underestimates the populations at higher levels. A non-linear fit as presented in Eq. (10) can fit the intensities of rotational levels as high as $J^{\prime}=9.5$, while reproducing the same temperature as the fit to the lowest three levels.

\subsection{Expansion to arbitrary waves}

So far, we have considered only a single wave with one given amplitude, wavelength and phase as an example. Different wave amplitudes $A_{\mathrm{w}}$ will, of course, change the populations seen in Fig. 4. In the limiting case, where $A_{\mathrm{w}}=0$, the original background temperature profile is obtained. Different wavelengths $\lambda_{w}$ will also change the shape of the atmospheric temperature profile. Short $\lambda_{\mathrm{w}}$ waves can change the temperature toward both higher and lower temperatures within the $\mathrm{OH}$ layer. Thus, their effect can be small when integrated over the whole layer. Longer $\lambda_{\mathrm{w}}$ waves, especially with $\lambda_{\mathrm{w}}$ on the order of, or longer than, the thickness of the $\mathrm{OH}$ layer, can introduce a temperature change that only warms one end of the layer and cools the opposite end. When such gradients reinforce the background temperature change with altitude, they can change the total integral over the $\mathrm{OH}$ layer substantially, as seen in Fig. 4. Lastly, the wave phase $\varphi_{\mathrm{w}}$ can change the influence of the wave on the total integral of the $\mathrm{OH}$ layer. The same wave would have the opposite temperature perturbation if $\varphi_{\mathrm{w}}$ was shifted by half a wavelength. In this case, the wave would work against the natural temperature gradient of the background atmosphere without a wave, resulting in a smaller effect than that shown in Fig. 4.

All three wave parameters $-A_{\mathrm{w}}, \lambda_{\mathrm{w}}$ and $\varphi_{\mathrm{w}}-$ should therefore be considered when modelling different waves to examine their influence on the total integrated $\mathrm{OH}$ spectrum. In this research, the three parameters were adjusted in equidistant steps. The amplitude $A_{\mathrm{w}}$ was varied between 0 and $40 \mathrm{~K}$ at an altitude of $90 \mathrm{~km}$, spanning a range of previously observed semi-diurnal tide amplitudes (Hagan et al., 1999; Oberheide et al., 2011; Picard et al., 2004; She et al., 2002; Shepherd and Fricke-Begemann, 2004; Zhang et al., 2006). The vertical wavelength $\lambda_{w}$ was varied between 2 and $80 \mathrm{~km}$, spanning the range of gravity waves, tides and planetary waves (Davis et al., 2013). The phase $\varphi_{\mathrm{w}}$ was varied between 0 and $2 \pi$.

\section{Results and discussion}

Figure 4 shows that a large atmospheric temperature gradient can produce higher populations in the higher $J^{\prime}$ rotational lines than would be expected from a strict LTE fit with only one effective temperature. We now want to quantify how large this apparent excess population can become and compare it to the study by Pendleton et al. (1993). An ensemble of waves was simulated as described above. The apparent excess population and the non-linearity parameter, $\beta$, were calculated for each wave-perturbed temperature background profile.

Figure 5 shows two different wave scenarios for the $(7,4)$ transition. The red plot (with the axis on the left-hand side) shows a no-wave scenario, where the atmospheric background temperature profile from the NRLMSISE-00 model is used. The apparent excess population is the ratio between the intensity of a rotational line integrated in altitude and the intensity of that line predicted by a Boltzmann distribution fitted to the distribution of integrated line intensities of the lowest three rotational lines using a single, effective temperature. Thus, an apparent excess population of 1 is the same population as that predicted from a single-temperature Boltzmann distribution fitted to the lowest three rotational lines. Similarly, an apparent excess population of 2 is a population twice as large as that predicted from this single-temperature Boltzmann distribution. It is clear that the first three lines with $J^{\prime} \leq 3.5$ can be characterized by a single, effective Boltzmann temperature. However, all higher lines show populations in excess of that expected from a single effective temperature. The effective overpopulation approaches approximately 1.12 for $J^{\prime}=9.5$, even though the $\mathrm{OH}$ molecule is thermalized with the local temperature at every altitude.

The blue bars (with the plot on the right side) show a scenario with a wave perturbing the atmosphere. This specific wave has an amplitude $A_{\mathrm{w}}=30 \mathrm{~K}$ at $90 \mathrm{~km}$ altitude, $\lambda_{\mathrm{w}}=30 \mathrm{~km}$ and $\varphi_{\mathrm{w}}=4.9 \mathrm{rad}$. This would be a large amplitude gravity wave but still within the range of atmospheric tidal observations. Although different $J^{\prime}$ levels are affected differently, the general shape is similar to that observed with the no-wave scenario. However, the magnitude of the effect is much greater. The first three lines show the populations expected in a single-temperature LTE case, while higher $J^{\prime}$ levels yield increasingly higher apparent excess populations. For the highest line considered in this paper with $J^{\prime}=9.5$, 

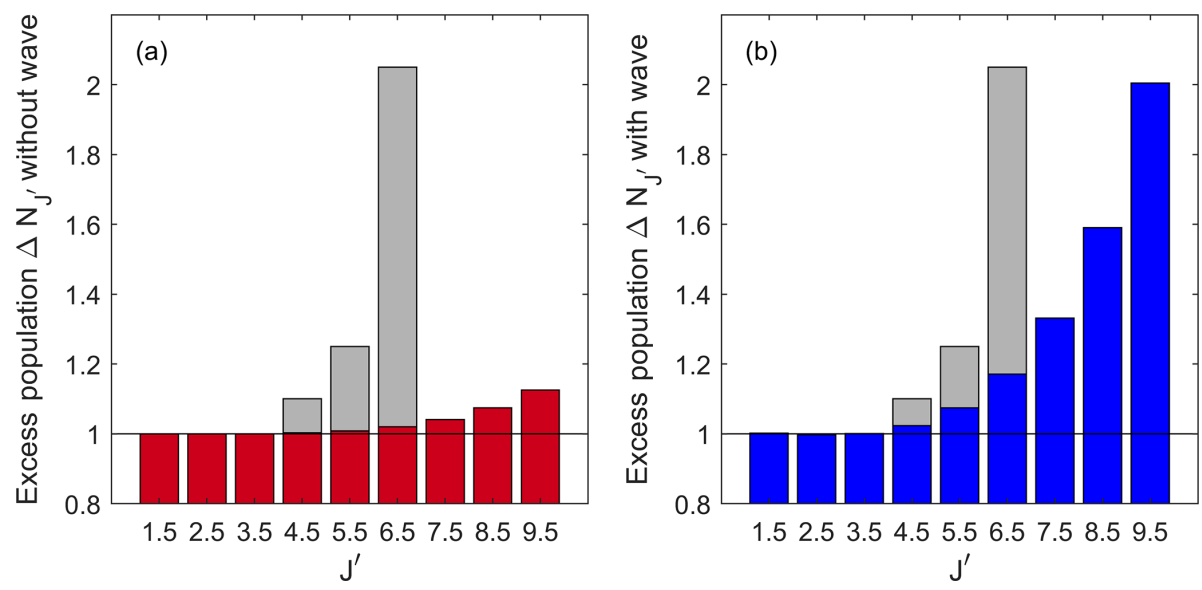

Figure 5. The calculated apparent excess population of the $\mathrm{OH}(7,4) P\left(J^{\prime}\right)$ lines relative to the Boltzmann population of the fitted temperature to the lowest three lines. Red in (a) is calculated with the climatological temperature gradient shown from NRLMSISE-00 above Boulder, Colorado $\left(40.0^{\circ} \mathrm{N} ; 105.6^{\circ} \mathrm{W}\right)$, in mid-July. Blue in $(\mathbf{b})$ is calculated with a wave of $A_{\mathrm{W}}=30 \mathrm{~K}$ at $90 \mathrm{~km}, \lambda_{\mathrm{W}}=30 \mathrm{~km}$ and a phase that yields the maximum effect, $\varphi_{\mathrm{W}}=4.9 \mathrm{rad}$. This is the same wave as presented in Figs. 3 and 4 . The distribution has a $\beta=0.019$. The grey bars represent the measurements ascribed to NLTE effects from Pendleton et al. (1993).
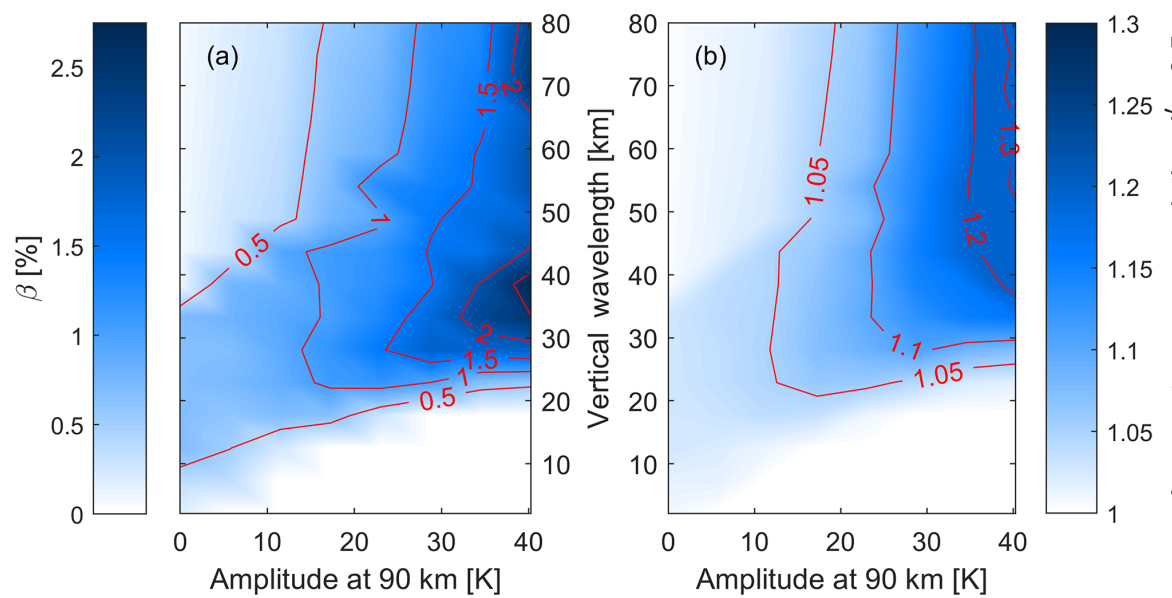

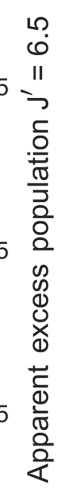

Figure 6. (a) The $\beta$ value from the non-linear fit (Eq. 10) and (b) the apparent excess population of $J^{\prime}=6.5(N=7)$ of the $(7,4)$ Meinel transition for the ensemble wave spectrum. The values are shown as a function of the wavelength, $\lambda_{\mathrm{w}}$, and the amplitude, $A_{\mathrm{W}}$. Each value represents the phase $\varphi_{\mathrm{w}}$ where the apparent excess population was largest. Extreme waves (tides) can show a non-linearity of about $2 \%$ corresponding to an apparent excess population of 1.3.

the apparent excess population is twice that expected for a Boltzmann distribution with a single effective temperature, despite the $\mathrm{OH}$ being in LTE for the rotational level populations at every altitude.

These results can be compared to the findings from Pendleton et al. (1993) in their Fig. 16 for the $(7,4)$ transition above Boulder, Colorado, during midsummer, the same season and transition as presented here. These results are shown as grey bars in Fig. 5a and b. Although the measurement from Pendleton et al. (1993) included NLTE effects, the overall shape of the distribution is similar to the LTE for the rotational level population simulations presented here. When comparing these results, note that Pendleton et al. (1993) use the lower-state quantum number $N$, which corresponds to our
$J^{\prime}+1 / 2$. The first three rotational levels in this study and in Pendleton et al. (1993) have populations that follow a Boltzmann distribution characterized by a single temperature, and above that, there is excess population. The difference between the absolute numbers we observe and those of Pendleton et al. (1993) indicates the portion of the NLTE that might be due to the temperature gradient effects observed here. For example, Pendleton et al. (1993) reported an apparent excess population of around a factor of 2 for the $P(N=7)$ (i.e. $\left.J^{\prime}=6.5\right)$ line. However, for the two cases presented here in Fig. 5, there is less apparent excess population. While the nowave scenario yields an apparent excess population of 1.02, a background profile with a wave yields 1.17 times the population. These numbers mean that up to about $17 \%$ of the effect 

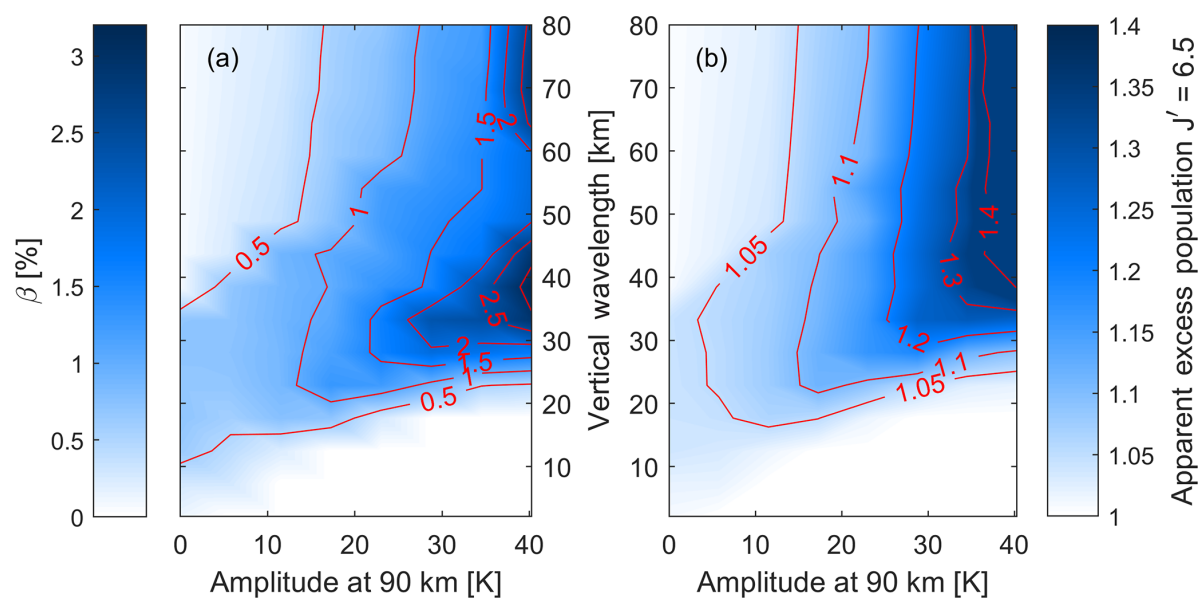

Figure 7. As Fig. 6 but this time for the $(3,1)$ transition. Both the $\beta$ value from the non-linear fit in (a) and the apparent excess population of the $J^{\prime}=6.5$ upper level in (b) show a stronger effect than seen for the $(7,4)$ transition in Fig. 6 .

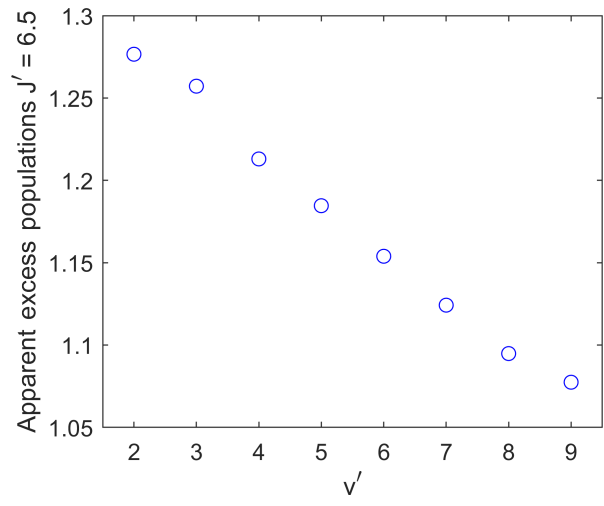

Figure 8. The maximum apparent excess population of the $J^{\prime}=6.5$ upper level for a wave with wavelength $30 \mathrm{~km}$ and an amplitude of $30 \mathrm{~K}$ at $90 \mathrm{~km}$ altitude as a function of the vibrational upper level. The effect of the waves creating the apparent excess population decreases with a rising vibrational quantum number $v^{\prime}$.

Pendleton et al. (1993) observed could be due to wave activity and not NLTE effects. Thus, the effect of the atmospheric temperature background has to be considered in addition to NLTE effects whenever the populations inferred from integrated airglow observations of high $J^{\prime}$ lines are to be used in kinetic thermalization studies.

This calculation of the apparent excess population can now be repeated for waves of different amplitudes, wavelengths and phases. Figure 6 shows the non-linearity in terms of the $\beta$ value of the temperature fit (from Eq. 10) and the corresponding apparent excess population of the sixth rotational line with $J^{\prime}=6.5(N=7)$, the highest line reported by Pendleton et al. (1993) in their NLTE study of the OH. The figure shows results related to the $(7,4)$ vibrational transition, which is the same transition that Pendleton et al. (1993) used. Figure $6 \mathrm{a}$ shows that for vertical wavelengths above about $20 \mathrm{~km}$, the non-linearity of the temperature fit increases with wave strength, approaching $2 \%$ of the linear temperature variation (see Eq. 10). The maximum non-linearity is observed at vertical wavelengths of about $20 \mathrm{~km}$ for waves with an amplitude below $10 \mathrm{~K}$, while for waves with an amplitude of $40 \mathrm{~K}$ the maximum non-linearity is observed at vertical wavelengths around $40 \mathrm{~km}$. For shorter vertical wavelengths, the non-linearity is smaller (at about $0.5 \%$ ) and decreases with increasing wave amplitude. Figure $6 \mathrm{~b}$ shows the apparent excess populations for a wave with a phase that yields the highest apparent excess population for a given wave amplitude and wavelength. There are small but observable effects in the limiting case of no waves (background atmosphere; see Fig. 5a), and the apparent excess population increases for longer wavelengths and stronger waves similarly to the nonlinearity of the fit. Extreme waves with $40 \mathrm{~K}$ amplitude at an altitude of $90 \mathrm{~km}$ can cause up to 1.3 apparent excess populations in the $J^{\prime}=6.5$ line.

While Fig. 6 shows the $(7,4)$ transition, Fig. 7 shows the same analysis for the commonly observed $(3,1)$ transition. Both the $\beta$ value and the apparent excess population look qualitatively similar to the $(7,4)$ band, but the effect of waves on the $(3,1)$ transition is about $30 \%$ stronger. That means that the non-linearity exceeds $2.5 \%$ and the apparent excess population of the $J^{\prime}=6.5$ level is up to 1.4 for the largest waves shown here.

Figures 6 and 7 show the results for the phase that created the largest apparent excess population. Hence, the phase is not constant for each point in these two figures but rather varies to show the largest apparent excess population for the wave amplitude and wavelength in question. The mean effect of all different phases simulated is independent of the transition and varies between $20 \%$ and $40 \%$ of the maximum effect presented in Figs. 6 and 7.

Tests showed that the difference in the apparent excess population between the $(3,1)$ and $(7,4)$ bands is unlikely to be due to the altitude separation of the different vibrational 
levels in the $\mathrm{OH}$ airglow layer (von Savigny et al., 2012). Repeating the analysis and weighting the $v^{\prime}=3$ and 7 levels with the same VER profile yields essentially the same result, as did performing the analysis in an unperturbed isothermal background temperature profile. Instead, the difference in the apparent excess population between the $(3,1)$ and $(7,4)$ bands is likely due to the compressed rotational energy structure of the higher vibrational levels that lie closer to the dissociation limit. This compression of the rotational energy levels is due to the increased moment of inertia and hence the reduced rotational constant, associated with the larger average inter-nuclear distance of the higher vibrational levels. Thus a given $J^{\prime}$ level in a low vibrational state will have more rotational energy than one in a high vibrational state. Thus, for a given temperature, higher rotational levels will be thermally populated in the higher vibrational levels. These thermally populated higher rotational levels then make the perturbing effects of waves relatively less important.

Figure 8 illustrates the dependency of the amount of apparent excess population on the different vibrational upper levels at different altitudes. For consistency, the apparent excess population of the $J^{\prime}=6.5$ upper level is again shown. All data points are for the wave presented above as an example, with an amplitude of $30 \mathrm{~K}$ at $90 \mathrm{~km}$ altitude and a vertical wavelength of $30 \mathrm{~km}$.

The smallest upper vibrational level of $v^{\prime}=2$ shows an apparent excess population of about 1.28, while the highest upper vibrational level of $v^{\prime}=9$ only shows an apparent excess population of about 1.08 .

\section{Conclusions}

Spectroscopic observations of the $\mathrm{OH}$ airglow have been commonly used to measure temperatures in the MLT. The $\mathrm{OH}$ radiates over an extended, Chapman-like layer that extends over several kilometres, over which the temperature is changing. Strong waves perturbing the MLT can make this change in temperature within the $\mathrm{OH}$ layer substantial. The simulations executed here show that these temperature profiles can create an apparent non-thermal population of the rotational levels in a given Meinel (v', v') band. Even though the simulations calculated the rotational population distribution of the $\mathrm{OH}$ to be in LTE with the surrounding gas at every altitude, the integrated intensities of the higher rotational lines indicate an apparent excess population that could be misinterpreted as contributing to the NLTE effects previously reported (Cosby and Slanger, 2007; Noll et al., 2015; Pendleton et al., 1993).

We have shown in this work that the influence of a wave with an amplitude of $30 \mathrm{~K}$ and a vertical wavelength of $30 \mathrm{~km}$ at an altitude of $90 \mathrm{~km}$ can produce an apparent excess population of 1.12; i.e. they can explain $12 \%$ of the effects previously ascribed to NLTE by Pendleton et al. (1993) for the $(7,4)$ transition. Larger waves cause an apparent excess pop- ulation of up to 1.3 and can therefore explain up to $30 \%$ of these effects. Smaller waves can also have an apparent excess population of up to 1.1 , and their impact cannot be ignored. Other transitions with lower vibrational quantum numbers show even higher apparent excess populations caused by this temperature-variation effect. We conclude that it is necessary to consider the temperature profile in order to infer $\mathrm{OH}$ rotational level population distributions from ground-based airglow observations.

Data availability. The SABER data presented in Fig. 1 are available under http://saber.gats-inc.com/ (last access: 8 January 2020). No other data were used in this paper.

Author contributions. CF modified the chemical model with dynamic inputs, ran the model and interpreted the model output. $\mathrm{CF}$ also wrote the paper. PJE supported the analysis process and the writing of the paper. REH gave input on the interpretation and the writing of the paper.

Competing interests. The authors declare that they have no conflict of interest.

Acknowledgements. This work was supported by the Research Council of Norway (CoE) under contract 223252/F50. We thank Halvor Borge, who undertook some preliminary studies on this research during his master thesis at NTNU in the spring semester of 2018. We also thank Kate Faloon for her work on the chemical model.

Financial support. This research has been supported by the Research Council of Norway (grant no. 223252/F50).

Review statement. This paper was edited by William Ward and reviewed by two anonymous referees.

\section{References}

Adler-Golden, S.: Kinetic parameters for $\mathrm{OH}$ nightglow modeling consistent with recent laboratory measurements, J. Geophys. Res.-Space, 102, 19969-19976, 1997.

Atkinson, R., Baulch, D. L., Cox, R. A., Crowley, J. N., Hampson, R. F., Hynes, R. G., Jenkin, M. E., Rossi, M. J., and Troe, J.: Evaluated kinetic and photochemical data for atmospheric chemistry: Volume I - gas phase reactions of $\mathrm{O}_{\mathrm{x}}, \mathrm{HO}_{\mathrm{x}}$, $\mathrm{NO}_{\mathrm{x}}$ and $\mathrm{SO}_{\mathrm{x}}$ species, Atmos. Chem. Phys., 4, 1461-1738, https://doi.org/10.5194/acp-4-1461-2004, 2004.

Baker, D. J. and Stair, J. A. T.: Rocket measurements of the altitude distributions of the hydroxyl airglow, Phys. Scripta, 37, 611-622, 1988 
Chalamala, B. R. and Copeland, R. A.: Collision dynamics of $\mathrm{OH}\left(\mathrm{X}^{2} \Pi, v=9\right)$, J. Chem. Phys., 99, 5807-5811, 1993.

Cosby, P. C. and Slanger, T. G.: OH spectroscopy and chemistry investigated with astronomical sky spectra, Can. J. Phys., 85, 7799, 2007.

Davis, R. N., Du, J., Smith, A. K., Ward, W. E., and Mitchell, N. J.: The diurnal and semidiurnal tides over Ascension Island $\left({ }^{\circ} \mathrm{S}, 14^{\circ} \mathrm{W}\right)$ and their interaction with the stratospheric quasi-biennial oscillation: studies with meteor radar, eCMAM and WACCM, Atmos. Chem. Phys., 13, 9543-9564, https://doi.org/10.5194/acp-13-9543-2013, 2013.

Dodd, J. A., Lipson, S. J., and Blumberg, W. A. M.: Formation and vibrational relaxation of $\mathrm{OH}\left(\mathrm{X}^{2} \Pi \mathrm{i}, \mathrm{v}\right)$ by $\mathrm{O}_{2}$ and $\mathrm{CO}_{2}$, J. Chem. Phys., 95, 5752-5762, 1991.

Dodd, J. A., Lipson, S. J., Lowell, J. R., Armstrong, P. S., Blumberg, W. A. M., Nadile, R. M., Adler-Golden, S. M., Marinelli, W. J., Holtzclaw, K. W., and Green, B. D.: Analysis of hydroxyl earthlimb airglow emissions: Kinetic model for state-to-state dynamics of $\mathrm{OH}(v, \mathrm{~N})$, J. Geophys. Res.-Atmos., 99, 3559-3585, 1994.

Dyer, M. J., Knutsen, K., and Copeland, R. A.: Energy transfer in the ground state of $\mathrm{OH}$ : Measurements of $\mathrm{OH}(v=8,10,11)$ removal, J. Chem. Phys., 107, 7809-7815, 1997.

Espy, P. J. and Hammond, M. R.: Atmospheric transmission coefficients for hydroxyl rotational lines used in rotational temperature determinations, J. Quant. Spectrosc. Ra., 54, 879-889, 1995.

Franzen, C., Hibbins, R. E., Espy, P. J., and Djupvik, A. A.: Optimizing hydroxyl airglow retrievals from long-slit astronomical spectroscopic observations, Atmos. Meas. Tech., 10, 3093-3101, https://doi.org/10.5194/amt-10-3093-2017, 2017.

French, W. J. R. and Mulligan, F. J.: Stability of temperatures from TIMED/SABER v1.07 (2002-2009) and Aura/MLS v2.2 (2004-2009) compared with OH(6-2) temperatures observed at Davis Station, Antarctica, Atmos. Chem. Phys., 10, 1143911446, https://doi.org/10.5194/acp-10-11439-2010, 2010.

Hagan, M. E., Burrage, M. D., Forbes, J. M., Hackney, J., Randel, W. J., and Zhang, X.: GSWM-98: Results for migrating solar tides, J. Geophys. Res.-Space, 104, 6813-6827, 1999.

Harrison, A. W., Llewellyn, E. J., and Nicholls, D. C.: Night airglow hydroxyl rotational temperatures, Can. J. Phys., 48, 1766-1768, 1970.

Harrison, A. W., Evans, W. F. J., and Llewellyn, E. J.: Study of the (4-1) and (5-2) Hydroxyl Bands in the Night Airglow, Can. J. Phys., 49, 2509-2517, 1971.

Herzberg, G.: Molecular Spectra and Molecular Structure. I. Spectra of the Diatomic Molecule, D. Van Nostrand Company, Inc., New York, 1950.

Holton, J. R.: The Role of Gravity Wave Induced Drag and Diffusion in the Momentum Budget of the Mesosphere, J. Atmos. Sci., 39, 791-799, 1982.

Kalogerakis, K. S.: Technical note: Bimodality in mesospheric $\mathrm{OH}$ rotational population distributions and implications for temperature measurements, Atmos. Chem. Phys., 19, 2629-2634, https://doi.org/10.5194/acp-19-2629-2019, 2019.

Kalogerakis, K. S., Matsiev, D., Cosby, P. C., Dodd, J. A., Falcinelli, S., Hedin, J., Kutepov, A. A., Noll, S., Panka, P. A., Romanescu, C., and Thiebaud, J. E.: New insights for mesospheric $\mathrm{OH}$ : multi-quantum vibrational relaxation as a driver for non- local thermodynamic equilibrium, Ann. Geophys., 36, 13-24, https://doi.org/10.5194/angeo-36-13-2018, 2018.

Knutsen, K., Dyer, M., and Copeland, R.: Collisional removal of $\mathrm{OH}(\mathrm{X} 2 \Pi, \nu=7)$ by $\mathrm{O}_{2}, \mathrm{~N}_{2}, \mathrm{CO}_{2}$, and $\mathrm{N}_{2} \mathrm{O}$, J. Chem. Phys., 104, 5798-5802, 1996.

Langhoff, S. R., Werner, H. J., and Rosmus, P.: Theoretical transition probabilities for the $\mathrm{OH}$ meinel system, I. Mol. Spectrosc., 118, 507-529, 1986.

Llewellyn, E. J., Long, B. H., and Solheim, B. H.: The Quenching of $\mathrm{OH}^{*}$ in the Atmosphere, Planet. Space Sci., 26, 525-531, https://doi.org/10.1016/0032-0633(78)90043-0, 1978.

Maylotte, D. H., Polanyi, J. C., and Woodall, K. B.: Energy Distribution Among Reaction Products. IV. $\mathrm{X}+\mathrm{HY}(\mathrm{X}=\mathrm{Cl}, \mathrm{Br} ; \mathrm{Y}=$ Br,I), Cl+DI, J. Chem. Phys., 57, 1547-1560, 1972.

McDade, I. C. and Llewellyn, E. J.: Kinetic parameters related to sources and sinks of vibrationally excited $\mathrm{OH}$ in the nightglow, J. Geophys. Res.-Space, 92, 7643-7650, 1987.

Mlynczak, M. G.: Energetics of the mesosphere and lower thermosphere and the SABER experiment, Adv. Space Res., 20, 11771183, 1997.

Mlynczak, M. G. and Solomon, S.: A detailed evaluation of the heating efficiency in the middle atmosphere, J. Geophys. Res.Atmos., 98, 10517-10541, 1993.

Noll, S., Kausch, W., Kimeswenger, S., Unterguggenberger, S., and Jones, A. M.: $\mathrm{OH}$ populations and temperatures from simultaneous spectroscopic observations of 25 bands, Atmos. Chem. Phys., 15, 3647-3669, https://doi.org/10.5194/acp-153647-2015, 2015.

Noll, S., Proxauf, B., Kausch, W., and Kimeswenger, S.: Mechanisms for varying non-LTE contributions to $\mathrm{OH}$ rotational temperatures from measurements and modelling. II. Kinetic model, J. Atmos. Sol.-Terr. Phys., 175, 100-119, 2018.

Oberheide, J., Forbes, J. M., Zhang, X., and Bruinsma, S. L.: Climatology of upward propagating diurnal and semidiurnal tides in the thermosphere, J. Geophys. Res.-Space, 116, https://doi.org/10.1029/2011ja016784, 2011.

Oliva, E., Origlia, L., Scuderi, S., Benatti, S., Carleo, I., Lapenna, E., and Pedani, M.: Lines and Continuum Sky Emission in the near Infrared: Observational Constraints from Deep High Spectral Resolution Spectra with Giano-Tng, A\&A, 581, A47, 2015.

Pendleton, W. R., Espy, P. J., Baker, D., Steed, A., Fetrow, M., and Henriksen, K.: Observation of $\mathrm{OH}$ Meinel $(7,4) \mathrm{P}(\mathrm{N}=13)$ transitions in the night airglow, J. Geophys. Res.-Space, 94, 505-510, 1989.

Pendleton, W. R., Espy, P. J., and Hammond, M. R.: Evidence for non-local-thermodynamic-equilibrium rotation in the $\mathrm{OH}$ nightglow, J. Geophys. Res.-Space, 98, 11567-11579, 1993.

Perminov, V. I., Semenov, A. I., and Shefov, N. N.: On rotational temperature of the hydroxyl emission, Geomagn. Aeronmy+, 47, 756-763, 2007.

Picard, R. H., Wintersteiner, P. P., Winick, J. R., Mertens, C. J., Mlynczak, M. G., Russell III, J. M., Gordley, L. L., Ward, W. E., She, C. Y., and O'Neil, R. R.: Tidal and layer structure in the mesosphere and lower thermosphere from TIMED/SABER CO2 15- $\mu$ m emission, SPIE, 2004.

Picone, J. M., Hedin, A. E., Drob, D. P., and Aikin, A. C.: NRLMSISE-00 empirical model of the atmosphere: Statistical comparisons and scientific issues, J. Geophys. Res.-Space, 107, 1468, https://doi.org/10.1029/2002JA009430, 2002. 
Polanyi, J. C. and Sloan, J. J.: Detailed rate constants for the reactions $\mathrm{H}+\mathrm{O}_{3} \rightarrow \mathrm{OH}\left(\right.$ nu', J') $+\mathrm{O}_{2}$ and $\mathrm{H}+\mathrm{NO}_{2} \rightarrow \mathrm{OH}\left(\mathrm{nu}, \mathrm{J}^{\prime}\right.$ ') + NO, United States, 1975-01-01 1975, 1975.

Polanyi, J. C. and Woodall, K. B.: Energy Distribution Among Reaction Products. VI. F+H2, D2, J. Chem. Phys., 57, 1574-1586, 1972.

Rothman, L. S., Gordon, I. E., Babikov, Y., Barbe, A., Chris Benner, D., Bernath, P. F., Birk, M., Bizzocchi, L., Boudon, V., Brown, L. R., Campargue, A., Chance, K., Cohen, E. A., Coudert, L. H., Devi, V. M., Drouin, B. J., Fayt, A., Flaud, J. M., Gamache, R. R., Harrison, J. J., Hartmann, J. M., Hill, C., Hodges, J. T., Jacquemart, D., Jolly, A., Lamouroux, J., Le Roy, R. J., Li, G., Long, D. A., Lyulin, O. M., Mackie, C. J., Massie, S. T., Mikhailenko, S., Müller, H. S. P., Naumenko, O. V., Nikitin, A. V., Orphal, J., Perevalov, V., Perrin, A., Polovtseva, E. R., Richard, C., Smith, M. A. H., Starikova, E., Sung, K., Tashkun, S., Tennyson, J., Toon, G. C., Tyuterev, V. G., and Wagner, G.: The HITRAN2012 molecular spectroscopic database, J. Quant. Spectrosc. Ra., 130, 4-50, 2013.

Russell, J. M., Mlynczak, M. G., Gordley, L. L., Tansock, J., and Esplin, R.: An overview of the SABER experiment and preliminary calibration results, in: Optical Spectroscopic Techniques and Instrumentation for Atmospheric and Space Research Iii, edited by: Larar, A. M., Proceedings of the Society of Photo-Optical Instrumentation Engineers (Spie), Spie-Int Soc Optical Engineering, Bellingham, 1999.

Sander, S. P., Friedl, R. R., Golden, D. M., Kurylo, M., Huie, R., Orkin, V., Moortgat, G., Ravishankara, A. R., Kolb, C. E., Molina, M., and Finlayson-Pitts, B.: Chemical Kinetics and Photochemical Data for Use in Atmosheric Studies, JPL Publication 02-25, 2003.

Sander, S. P., Friedl, R. R., DeMore, W. B., Ravishankara, A. R., and Kolb, C. E.: Chemical Kinetics and Photochemical Data for Use in Stratospheric Modeling Supplement to Evaluation 12: Update of Key Reactions, 2019.
She, C. Y., Chen, S., Williams, B. P., Hu, Z., Krueger, D. A., and Hagan, M. E.: Tides in the mesopause region over Fort Collins, Colorado $\left(41^{\circ} \mathrm{N}, 105^{\circ} \mathrm{W}\right)$ based on lidar temperature observations covering full diurnal cycles, J. Geophys. Res.-Atmos., 107, ACL 4-1-ACL 4-12, 2002.

Shepherd, M. and Fricke-Begemann, C.: Study of the tidal variations in mesospheric temperature at low and mid latitudes from WINDII and potassium lidar observations, Ann. Geophys., 22, 1513-1528, https://doi.org/10.5194/angeo-22-1513-2004, 2004.

Sivjee, G. G. and Hamwey, R. M.: Temperature and chemistry of the polar mesopause OH, J. Geophys. Res.-Space, 92, 4663-4672, 1987.

Sivjee, G. G., Dick, K. A., and Feldman, P. D.: Temporal variations in night-time hydroxyl rotational temperature, Planet. Space Sci., 20, 261-269, 1972.

Smith, S. M., Baumgardner, J., Mertens, C. J., Russell, J. M., Mlynczak, M. G., and Mendillo, M.: Mesospheric OH temperatures: Simultaneous ground-based and SABER OH measurements over Millstone Hill, Adv. Space Res., 45, 239-246, 2010.

Vincent, R. A.: Gravity-wave motions in the mesosphere, J. Atmos. Terr. Phys., 46, 119-128, 1984.

von Savigny, C., McDade, I. C., Eichmann, K.-U., and Burrows, J. P.: On the dependence of the $\mathrm{OH}^{*}$ Meinel emission altitude on vibrational level: SCIAMACHY observations and model simulations, Atmos. Chem. Phys., 12, 8813-8828, https://doi.org/10.5194/acp-12-8813-2012, 2012.

Xu, J., Gao, H., Smith, A. K., and Zhu, Y.: Using TIMED/SABER nightglow observations to investigate hydroxyl emission mechanisms in the mesopause region, J. Geophys. Res.-Atmos., 117, 1-22, 2012.

Zhang, X., Forbes, J. M., Hagan, M. E., Russell III, J. M., Palo, S. E., Mertens, C. J., and Mlynczak, M. G.: Monthly tidal temperatures 20-120 km from TIMED/SABER, J. Geophys. Res.-Space, 111, 0148-0227, https://doi.org/10.1029/2005ja011504, 2006. 